\title{
Enhancing research publications and advancing scientific writing in health research collaborations: sharing lessons learnt from the trenches
}

This article was published in the following Dove Press journal: Journal of Multidisciplinary Healthcare

\author{
Guowei $\mathrm{Li}^{1-3}$ \\ Yanling Jin' \\ Lawrence Mbuagbaw ${ }^{1,2}$ \\ Lisa Dolovich ${ }^{1,2,4}$ \\ Jonathan D Adachi ${ }^{2,5}$ \\ Mitchell AH Levine ${ }^{1-3,5}$ \\ Deborah Cook ${ }^{1,2,5}$ \\ Zainab Samaan ${ }^{1,2}$ \\ Lehana Thabane ${ }^{1,2}$ \\ 'Department of Health Research \\ Methods, Impact and Evidence, \\ McMaster University, Hamilton, ON, \\ Canada; ${ }^{2}$ St. Joseph's Healthcare \\ Hamilton, McMaster University, \\ Hamilton, ON, Canada; ${ }^{3}$ Centre for \\ Evaluation of Medicines, Programs for \\ Assessment of Technology in Health \\ (PATH) Research Institute, McMaster \\ University, Hamilton, ON, Canada; \\ ${ }^{4}$ Department of Family Medicine, \\ McMaster University, Hamilton, ON, \\ Canada; ${ }^{5}$ Department of Medicine, \\ McMaster University, Hamilton, ON, \\ Canada
}

Correspondence: Guowei Li Department of Health Research Methods, Impact and Evidence, McMaster University, 50I-25 Charlton Avenue East, Hamilton, ON, Canada L8N IY2

$\mathrm{Tel}+\mathrm{I} 9059621068$

Fax + I $90552 \mid 1297$

Email lig28@mcmaster.ca
Background: Disseminating research protocols, processes, methods or findings via peerreviewed publications has substantive merits and benefits to various stakeholders.

Purpose: In this article, we share strategies to enhance research publication contents (ie, what to write about) and to facilitate scientific writing (ie, how to write) in health research collaborations.

Methods: Empirical experience sharing.

Results: To enhance research publication contents, we encourage identifying appropriate opportunities for publications, publishing protocols ahead of results papers, seeking publications related to methodological issues, considering justified secondary analyses, and sharing academic process or experience. To advance writing, we suggest setting up scientific writing as a goal, seeking an appropriate mentorship, making full use of scientific meetings and presentations, taking some necessary formal training in areas such as effective communication and time and stress management, and embracing the iterative process of writing.

Conclusion: All the strategies we share are dependent upon each other; and they advocate gradual academic accomplishments through study and training in a "success-breeds-success" way. It is expected that the foregoing shared strategies in this paper, together with other previous guidance articles, can assist one with enhancing research publications, and eventually one's academic success in health research collaborations.

Keywords: research publication, scientific writing, health research collaboration

\section{Background}

In health research, one of the most important bibliometrics in research productivity is the number of publications per researcher. ${ }^{1}$ By publishing research, researchers can share their findings with the international scientific community, meet the requirements of public accountability, accomplish a successful academic career, and attract more funding for further research. ${ }^{2}$ Moreover, disseminating research protocols, processes, methods, or findings via peer-reviewed publications has significant merits and benefits to various stakeholders including researchers, practitioners or healthcare providers, policy makers, patients, industry, students or trainees, and so on. For instance, peerreviewed publications are routinely used in academic institutions as a metric for research productivity in promoting individual researchers. Research publications can provide healthcare providers with the latest or best available evidence to support their clinical practice decisions. ${ }^{3}$ Besides, peer-reviewed publications may also aid in decision-making process for policy makers by providing scientific key messages and assist patients in self-education and health promotion. ${ }^{4}$ For students or trainees, the publication process 
can help them to demonstrate their understanding of research methodology, refine writing skills, build up confidence in decision-making, and learn from constructive feedback from reviewers. ${ }^{5,6}$ In addition, publications can launch the careers of students or trainees and give them exposure to potential employers who read their work. In contrast, for example, failure to publish findings from a clinical trial may unethically expose patients to hazards without providing benefits. ${ }^{7}$ Therefore, in this study, we aimed to share our empirical experience and provide some general and practical strategies to 1) enhance research publication contents (ie, what to write about) in health research collaborations and 2) facilitate the scientific writing process (ie, how to write). We expect that the shared strategies may assist researchers with increasing their research output and eventually their academic success in health research collaborations.

\section{Strategies for enhancing research publication contents (ie, what to write about) Identifying appropriate opportunities of publications or presentations for each study}

Before designing a health research study, researchers are usually encouraged to search the literature and appraise the current evidence in order to support the feasibility and necessity of the planned study. Summarizing the evidence can generally yield review articles (such as systematic reviews, scoping or narrative reviews), perspectives, brief reports, or commentaries. This literature search may sometimes require a registration or protocol to design the search strategy; as detailed below, publishing protocols before the results paper for such practices gains significant benefits, which consequently assists in enhancing research publications.

Furthermore, presenting research findings at a professional meeting including research rounds in department or faculty and at national or international meeting could also potentially produce publications such as abstracts, research updates, and conference archives. All these appropriate attempts and opportunities can enhance the quality and quantity of the research output as well. Of note, keep in mind no duplication of work can be published (except for conference abstracts) as separate papers, and authors need to be cautious of plagiarizing publications from their previous work. Nevertheless, in some instances, important messages can be disseminated through publishing summaries of the work that has been published already. For instance, the CONSORT guidance (Consolidated Standards of Report- ing Trials; http://www.consort-statement.org/) is published in different journals to aid in reporting clinical trials; and results of Cochrane reviews are often published in abridged format to help disseminate the findings.

\section{Planning a study is expected to yield at least one publication}

Turning a research question into a study generally begins with a detailed protocol or study registration. Although some registries are available to present the key elements for the ongoing studies (eg, clinicaltrials.gov for clinical trials and PROSPERO for systematic reviews), this initial stage of planning a study can also usually produce at least one publication of protocol paper. A protocol paper is generally defined as the original research plan with detailed description of objective(s), study subjects or participants, outcomes, study design, methodology, statistical plan, and other related information that are identified prior to any study results. Publishing protocols can achieve multiple benefits including 1) obtaining feedback through peer review on the study plan; 2) demonstrating public and transparent declaration of the study (eg, patients can search the Internet and keep updated with what studies are in progress; readers can compare the original intent [protocol] with what is actually done [final report]; other researchers, funders, and decisionmakers can see what studies are ongoing to determine optimal resource allocation and avoid unnecessary research duplicates; systematic reviewers can locate eligible ongoing studies; investigators can seek networking opportunities with other researchers; etc); 3 ) encouraging publication of either positive or negative results, which may potentially minimize publication bias; 4) allowing more details on explanation and discussion of methodological choices to be published so that the results paper can focus on study results and their interpretations; and 5) enhancing publication track record for researchers. For instance, we have blogged about the importance of prospective publication of protocols for pilot and feasibility studies. ${ }^{8}$

Protocols followed by results papers can be found in different study designs in health research. Examples for randomized controlled trials (RCTs) include the singlecentered CAMPS (Cameroon Mobile Phone Short Message Service) trial that explored the effect of mobile phone text messaging versus usual care on adherence to highly active anti-retroviral therapy (ART) in HIV-positive adults ${ }^{9,10}$ and the PROTECT (Prophylaxis for Thromboembolism in Critical Care Trial) that compared the effect of dalteparin versus unfractionated heparin on venous thromboembolism in critically ill patients ${ }^{11,12}$ (Table 1). Likewise, examples of 
Table I Examples of studies with published protocols and results papers

\begin{tabular}{|c|c|c|c|}
\hline Study design & Study name & Protocol paper* & Results paper* \\
\hline \multirow{2}{*}{ Randomized controlled trials } & CAMPS trial & Mbuagbaw, $2011^{9}$ & Mbuagbaw, $2012^{10}$ \\
\hline & PROTECT & Cook, 20II"I & Cook, $2011^{12}$ \\
\hline \multirow[t]{2}{*}{ Observational studies } & TOMIS III & Sword, $2009^{13}$ & Sword, $201 \mathrm{I}^{14}$ \\
\hline & $\mathrm{N} / \mathrm{A}$ & $\mathrm{Li}, 2015^{15}$ & $\mathrm{Li}, 2016^{16}$ \\
\hline \multirow[t]{2}{*}{ Systematic reviews and meta-analyses } & N/A & Morfaw, $2012^{17}$ & Morfaw, $2013^{18}$ \\
\hline & N/A & $\mathrm{Li}, 2013^{19}$ & $\mathrm{Li}, 2014^{20}$ \\
\hline Individual patient data meta-analysis & $\begin{array}{l}\text { N/A } \\
\text { OA Trial Bank study }\end{array}$ & $\begin{array}{l}\text { Mbuagbaw, } 201 \mathrm{I}^{21} \\
\text { van Middelkoop, } 2013^{23}\end{array}$ & $\begin{array}{l}\text { Mbuagbaw, 201222 } \\
\text { van Middelkoop, } 2016^{24}\end{array}$ \\
\hline
\end{tabular}

Note: *Expressed as first author, publication year reference number.

Abbreviations: CAMPS trial, Cameroon Mobile Phone Short Message Service trial; PROTECT, Prophylaxis for Thromboembolism in Critical Care Trial; TOMIS III, The Ontario Mother and Infant Study III; OA Trial Bank, Osteoarthritis Trial Bank study; N/A, not applicable.

observational studies ${ }^{13-16}$ and systematic reviews or (network) meta-analyses ${ }^{17-20}$ are also summarized in Table 1 . In addition, meta-analyses using individual patient data (IPD) can also yield publications for their protocols and results. Examples can be found in an IPD meta-analysis of three RCTs assessing mobile phone text messages for improving adherence to ART ${ }^{21,22}$ and the osteoarthritis trial bank study using data from existing trials to evaluate the effect of intra-articular glucocorticoids on knee or hip osteoarthritis in patients with severe pain and inflammatory signs. ${ }^{23,24}$ Similarly, publishing protocols followed by results can also include mixed methods research studies, pilot RCTs, reporting guideline development, quality of reporting assessment for published studies, and statistical analysis plans, among others (examples shown in Table S1).

\section{Seeking opportunities to lead a methodological paper for each study}

Methodological issues in a study are always worth one's investigation and efforts, because they are usually related to the validity of the study findings. Seeking opportunities to lead a methodological paper for the study is an important way of enhancing research publications. For instance, in the original report from the PROTECT, no difference in proximal leg deep vein thromboses (PLDVT) between dalteparin versus unfractionated heparin was found using a standard survival analysis. ${ }^{12}$ Given the presence of competing risk of death (ie, death prior to a PLDVT precluded the occurrence of subsequent PLDVT) for the critically ill patients who were at high risk of death, a competing risk analysis was conducted to evaluate the impact of the competing risk on treatment effect estimates, which was then found to be in agreement with the results from the original report $^{25}$ (Table 2). As shown in Table 2, other methodological publication examples include 1) adjusting for center effects in multicenter RCTs; ${ }^{26}$ 2) adjusting for clustering in cluster ran- domization trials in analyses; ${ }^{27}$ and 3 ) dealing with composite outcomes in trials, ${ }^{28}$ among others.

Guideline development, including the reporting guidance and the reporting quality assessment, can also lead to methodological publications. A typical example can be found in the CONSORT statement and its extensions for RCTs regarding the reporting of clinical trials. Other methodological publications from reporting guideline statements and explanations or elaborations can be referred to the EQUATOR (Enhancing the QUAlity and Transparency of health Research; http://www. equator-network.org/) network. Subsequently, methodological studies can be conducted to assess the quality of reporting or adherence to reporting guidelines ${ }^{29}$ or to investigate the relationship between application of guidelines and improved quality of reporting ${ }^{30}$ (Table 2 ).

\section{Considering justified secondary analyses to make full use of the data}

Conducting a study is usually time-consuming and costly; therefore, it is not uncommon to employ the data already collected to answer other research questions and run secondary analyses. In fact, making full use of the data via justified secondary analyses is a frequently encountered practice in the literature for clinical trials as well as observational studies. Taking PROTECT as an example again, except for its main purpose of comparing dalteparin with unfractionated heparin on PLDVT, ${ }^{11,12}$ subsequent secondary analyses yielded multiple publications including building a prediction model for all-cause death, ${ }^{31}$ exploring the risk factors for death in a subgroup of patients who were admitted to intensive care units with a primary diagnosis of pneumonia ${ }^{32}$ and looking at the predictors and consequences of co-enrollment of critically ill patients, ${ }^{33}$ among others.

Likewise, the observational research of GLOW (Global Longitudinal Study of Osteoporosis in Women) was 
Table 2 Examples of using some strategies to enhance research publication contents

\begin{tabular}{|c|c|}
\hline Strategies & Example study reference* \\
\hline \multicolumn{2}{|l|}{ Exploring methodological issues in studies } \\
\hline Competing risk analysis & $\mathrm{Li}, 2015^{25}$ \\
\hline Adjusting for centre effects in multicentre trials & Kahan, $2013^{26}$ \\
\hline Adjusting for clustering in cluster randomization trials & Ma, $2009^{27}$ \\
\hline Dealing with composite outcomes in trials & Pogue, $2012^{28}$ \\
\hline \multirow[t]{2}{*}{ Guideline development } & CONSORT statement ${ }^{\#}$ \\
\hline & EQUATOR network ${ }^{\#}$ \\
\hline \multirow[t]{2}{*}{ Quality of reporting } & Samaan, $2013^{29}$ \\
\hline & Tunis, $2013^{30}$ \\
\hline \multicolumn{2}{|l|}{ Performing multiple secondary analyses using data already collected } \\
\hline \multirow[t]{4}{*}{ Studies based on trial data } & Publications using PROTECT data: \\
\hline & $\mathrm{Li}, 2016^{31}$ \\
\hline & $\mathrm{Li}, 2016^{32}$ \\
\hline & Cook, $2013^{33}$ \\
\hline \multirow[t]{5}{*}{ Studies based on observational study data } & Publications using GLOW data: \\
\hline & $\mathrm{Li}, 2014^{35}$ \\
\hline & $\mathrm{Li}, 2015^{36}$ \\
\hline & $\mathrm{Li}, 2015^{37}$ \\
\hline & $\mathrm{Li}, 2016^{38}$ \\
\hline \multicolumn{2}{|l|}{ Sharing academic process or experience } \\
\hline Experiences in mentorship relationships & Mbuagbaw, $2013^{39}$ \\
\hline Development of new courses and promote teaching & Thabane, $2008^{40}$ \\
\hline Promoting the teaching in courses & Pullenayegum, $2009^{41}$ \\
\hline Capacity building and skill development & Thabane, $201 I^{42}$ \\
\hline Challenges, lessons, or suggestions during research or collaborations & Thabane, $2010^{43}$ \\
\hline
\end{tabular}

Notes: *Expressed as first author, publication yearreference number. "CONSORT statement, Consolidated Standards of Reporting Trials statement. Available from: http://www. consort-statement.org/;51 EQUATOR network, Enhancing the QUAlity and Transparency of health Research network. Available from http://www.equator-network.org/.52 Abbreviations: PROTECT, Prophylaxis for Thromboembolism in Critical Care Trial; GLOW, Global Longitudinal Study of Osteoporosis in Women.

originally performed to evaluate the health consequences and risk factors for fragility fractures in women aged no less than 55 years. ${ }^{34}$ We used the GLOW Hamilton cohort to construct a frailty index (FI) of deficit accumulation to measure frailty in the women, ${ }^{35}$ compare the predictive accuracy between the FI and phenotypic model and FRAX (fracture risk assessment tool) in risks of adverse health outcomes, ${ }^{36,37}$ and assess the relationship between a major osteoporotic fracture and frailty change measured by the FI. ${ }^{38}$ These multiple secondary analyses fully employed the existing data collected from the original study, which was a significantly useful, cost- and time-effective, and practical approach to enhancing research publications in health research collaborations. However, before attempting such secondary analyses, one needs to be mindful of the initial purpose of data collection, patient consent, and related ethics approvals when applicable.

\section{Sharing academic process or experience}

The academic process in teaching or conducting health research may provide some insights and suggestions for other researchers. It is therefore worth sharing what the common difficulties would be, how they can be solved, and what lessons or experiences can be gained during the teaching or research activities.

For example, during our teaching process, we have shared our suggestions about how to construct an efficient mentormentee relationship with junior researchers, ${ }^{39}$ how to develop a health collaboration course, ${ }^{40}$ how to promote teaching, ${ }^{41}$ and how to help build up students' or mentees' capacity and skills in health research. ${ }^{42}$ Similarly, we have published some studies focusing on the challenges, lessons, and suggestions during our research practice or collaborations with other researchers. These include a guidance on how to pose a research question, commentaries on how to conduct a trial with limited resources, how to handle data withdrawal, and tutorials on pilot studies, sensitivity analyses, and multiplicity issues in clinical trials (details shown in Table S2). Of note, sharing academic process and writing about lessons gained in teaching and research can usually have major impact and important contributions. For instance, one of our tutorials published ${ }^{43}$ has been highly accessed and cited in the literature and continue to be so. 


\section{Strategies for facilitating scientific writing (ie, how to write) Setting up scientific writing as a career goal}

It is always important for one to set up career goals for what they aim to achieve in the near or long-term future. Achieving a predetermined plan can always bring one motivation and satisfactions, which would in turn encourage one to accomplish consequent goals. We would refer readers to Sackett's publications about how to make scientific writing and research publications as parts of career goals and how to realize these goals. ${ }^{44}$ For example, Sackett mentioned three determinants of academic success as a clinician-scientist including seeking mentorship, making a priority checklist, and having good time management skills. ${ }^{44}$ Mentorship is discussed in the next section. In his other publication, he elaborated on the priority setting for academic success and generated four lists. ${ }^{45}$ The lists included:

List \#1: Things you are doing now that you want to quit.

List \#1a: Things you have just been asked to do that you want to refuse to do.

List \#2: Things you are not doing that you want to start doing.

List \#3: Things you are doing that you want to continue doing.

List \#4: Strategies for improving the balance within your lists by shortening Lists \#1 and \#1a (quit and refuse) and lengthening List \#2 (start) over the next 6 months. ${ }^{45}$

Achieving balance by titrating Lists \#2 (start) and \#3 (continue) against Lists \#1 and \#1a (quit and refuse), as well as improving the balance by List \#4, is crucial for the realization of a set of research, teaching, and clinical activities in one's life-long career. ${ }^{45}$ Besides, improving time management skills including ruthlessly protecting time for writing is obviously essential to academic success. ${ }^{45}$ Collectively, all these three elements can be substantially useful for facilitating writing in health collaborations and consequently for realizing the anticipated goals of getting research publications.

\section{Seeking an appropriate mentorship}

For students or trainees, getting a mentor is very important to improving their writing skills and achieving academic accomplishments. Getting mentored can help mentees to guarantee research time and resources, facilitate writing process, improve writing skills, and aid in more publications and research funding as part of the anticipated research goals. ${ }^{46}$ An appropriate mentorship also provides one with career and personal advice, protects one against unnecessary academic buffeting and bad behavior of other academics, offers more academic opportunities, and accelerates the acquisition of important career skills for mentees. ${ }^{47}$

\section{Making full use of scientific meetings and presentations}

Targeting a professional scientific meeting and submitting an abstract for an oral or poster presentation can provide one with a unique opportunity to facilitate the writing. The specific deadline for the meeting or presentation requires one to complete the product of at least an abstract, which is essentially the first step toward a publication. Besides, the oral or poster presentation usually shapes a structured outline for the publication as a very important part of the writing process. ${ }^{48}$ Moreover, feedback from the audience, as well as discussion during the meeting, is significantly helpful with the actual writing especially for the "Discussion" section in a publication. We encourage researchers to make notes of key information and comments from the meeting and presentation before leaving the meeting. In fact one may even complete the first draft for that publication by using the remaining time wisely after delivering the presentation and attending only some sessions of interest in the meeting (good time management skills).

\section{Taking formal training in key areas to improve the overall effectiveness in writing}

Without a doubt, we believe that one would benefit from taking some formal training or courses on time management, stress management, improving scientific writing, and effective communication. For instance, during training to improve scientific writing, the practice of writing, the requirement of regular literature reading, and the feedback from the instructors will facilitate one's writing skills and build up confidence in continuing writing ${ }^{49}$ Furthermore, apparently the soft skills gained from the courses and training would accelerate not only the writing process and subsequent research publications but also other accomplishments in their academic career such as networking, future research collaborations, and peer support.

\section{Embracing the iterative process of writing to improve the quality of publication}

The process of writing involves an iterative process whereby an author generates an outline or first draft and then refines 
the draft over time. The outline and subsequent drafts will often benefit from discussion with and input from other members of the study team and from the opportunity to set the paper aside for a short period of time and then review it with a fresh perspective. Most publications in health sciences are authored by more than one person. This provides the opportunity to build on the collective wisdom of the team to facilitate scientific writing and improve the quality of publication.

\section{Conclusion}

In this paper, we have proposed several strategies for what to write about and how to write to enhance research publications in health collaborations. Each of us has used the strategies in varying degrees of deliberateness or planning and achieved success in mentoring students and trainees. We encourage identifying appropriate opportunities of publications, publishing protocols ahead of results papers, seeking publications related to methodological issues, considering justified secondary analyses, and sharing academic process or experience. To advance scientific writing, we suggest setting up scientific writing as a goal, seeking an appropriate mentorship, making full use of scientific meetings and presentations, taking some necessary formal training in areas such as effective communication and time and stress management, and embracing the iterative process of writing. These strategies we shared so far are practically applicable to our students and trainees. For instance, empirically all our $\mathrm{PhD}$ students take 2-4 years to complete their study with at least three to six publications prior to defending their thesis.

All the strategies are dependent upon one another; and they advocate gradual academic accomplishments through study and training in a "success-breeds-success" way. For example, publishing a protocol can generally help build up one's confidence in writing results paper, pursuing more projects, presenting at a professional meeting, sharing academic experience, and improving time management skills, among others. Therefore, we would strongly recommend that students, trainees, and novice researchers should pursue all possible and appropriate attempts at publications and actual writing.

There are some articles about suggestions on how to accelerate publishing research. For instance, we would refer readers to some guidance papers ${ }^{2,50}$ as well as a collection of scientific writing tips series (http://www.jclinepi.com/content/jce-Writing-Tips-Series). These previous publications give a detailed guidance on 1) how to achieve high-quality writing including the sections of title and abstract, introduc- tion, methods, results, discussion, tables and figures, and references and 2) how to advance all the processes of publishing a paper including authorship identification, choosing a target journal, manuscript submission, responding reviewers' comments, and dealing with the editors. Our paper largely agrees with the tips for advancing the actual writing and submission processes. However, unlike their step-by-step suggestions on how to write and publish a specific publication, our paper may also provide some novel insight into how to locate the publication contents (ie, what to write about) and how to employ available resources for long-term efficient writing (ie, how to write) during one's academic career. It is expected that our paper, together with the previous guidance articles, may assist readers in their improved writing and research output.

However, in this paper, we do not focus on the impact of research publications, because our goal is to assist others with increasing their own publication output and advancing their scientific writing in health research collaborations. Likewise, no empirical experience on how to play an appropriate role in a team as a collaborator and a co-author is provided in this paper. It would be a worthwhile endeavor to share strategies to improve the impact of research publications and collaborate with other researchers with multidisciplinary background in the future.

In summary, research publications bring substantial benefits and merits. We hope the foregoing shared strategies, together with other previous guidance papers, can help others in enhancing their research publications and eventually their academic success.

\section{Acknowledgment}

We acknowledge Dr Sharon Straus for her helpful suggestion and input in the manuscript.

\section{Author contributions}

All authors contributed toward data analysis, drafting and critically revising the paper and agree to be accountable for all aspects of the work.

\section{Disclosure}

The authors report no conflicts of interest in this work.

\section{References}

1. Abramo G, D'Angelo CA. How do you define and measure research productivity? Scientometrics. 2014;101:1129-1144.

2. Knottnerus JA, Tugwell P. Communicating research to the peers. J Clin Epidemiol. 2007;60:645-647.

3. Evidence-Based Medicine Working Group. Evidence-based medicine. A new approach to teaching the practice of medicine. JAMA. 1992;268:2420-2425. 
4. Pagani F. Peer review as a tool for co-operation and change: an analysis of an OECD working method. Afr Secur Rev. 2002;11:15-24.

5. Dickersin K, Chalmers I. Recognizing, investigating and dealing with incomplete and biased reporting of clinical research: from Francis Bacon to the WHO. J R Soc Med. 2011;104:532-538.

6. Brownlow S. Going the extra mile. The rewards of publishing your undergraduate research. Psi Chi J Undergrad Res. 1997;2:83-85.

7. Glasziou P, Altman DG, Bossuyt P, et al. Reducing waste from incomplete or unusable reports of biomedical research. Lancet 2014;383:267-276.

8. BioMed Central Blog network. Prospective publication of protocols and results of pilot and feasibility works. Available from: http://blogs. biomedcentral.com/on-medicine/announcement/prospective-publication-of-protocols-and-results-of-pilot-and-feasibility-works/?utm campaign=shareaholic\&utm_medium=email_this\&utm_source=email. Accessed August 2016.

9. Mbuagbaw L, Thabane L, Ongolo-Zogo P, et al. The Cameroon Mobile Phone SMS (CAMPS) trial: a protocol for a randomized controlled trial of mobile phone text messaging versus usual care for improving adherence to highly active anti-retroviral therapy. Trials. 2011; 12:5.

10. Mbuagbaw L, Thabane L, Ongolo-Zogo P, et al. The Cameroon Mobile Phone SMS (CAMPS) trial: a randomized trial of text messaging versus usual care for adherence to antiretroviral therapy. PLoS One. 2012;7:e46909.

11. Cook D, Meade M, Guyatt G, et al. PROphylaxis for ThromboEmbolism in Critical Care Trial protocol and analysis plan. Journal of Critical Care. 2011;26:223. e221-e223. e229.

12. Cook D, Meade M, Guyatt G, et al. Dalteparin versus unfractionated heparin in critically ill patients. $N$ Engl J Med. 2011;364: 1305-1314.

13. Sword W, Watt S, Krueger P, et al. The Ontario Mother and Infant Study (TOMIS) III: a multi-site cohort study of the impact of delivery method on health, service use, and costs of care in the first postpartum year. BMC Pregnancy Childbirth. 2009;9:16.

14. Sword W, Kurtz Landy C, Thabane L, et al. Is mode of delivery associated with postpartum depression at 6 weeks: a prospective cohort study. BJOG 2011;118:966-977.

15. Li G, Holbrook A, Delate T, Witt DM, Levine MA, Thabane L. Prediction of individual combined benefit and harm for patients with atrial fibrillation considering warfarin therapy: a study protocol. BMJ Open. 2015;5:e09518.

16. Li G, Thabane L, Delate T, et al. Can we predict individual combined benefit and harm of therapy? Warfarin therapy for atrial fibrillation as a test case. PLoS One. 2016;11:e0160713.

17. Morfaw FL, Thabane L, Mbuagbaw LC, Nana PN. Male participation in prevention programmes of mother to child transmission of HIV: a protocol for a systematic review to identify barriers, facilitators and reported interventions. Syst Rev. 2012;1:13.

18. Morfaw F, Mbuagbaw L, Thabane L, et al. Male involvement in prevention programs of mother to child transmission of HIV: a systematic review to identify barriers and facilitators. Syst Rev. 2013;2:1.

19. Li G, Zhang Y, Mbuagbaw L, Holbrook A, Levine MA, Thabane L. Effect of green tea supplementation on blood pressure among overweight and obese adults: a protocol for a systematic review. BMJ Open. 2014;4:e04971.

20. Li G, Zhang Y, Thabane L, et al. Effect of green tea supplementation on blood pressure among overweight and obese adults: a systematic review and meta-analysis. J Hypertens. 2015;33:243-254.

21. Mbuagbaw L, van der Kop ML, Lester RT, et al. Mobile phone text messages for improving adherence to antiretroviral therapy (ART): a protocol for an individual patient data meta-analysis of randomised trials. BMJ Open. 2013;3:e002954.

22. Mbuagbaw L, van der Kop ML, Lester RT, et al. Mobile phone text messages for improving adherence to antiretroviral therapy (ART): an individual patient data meta-analysis of randomised trials. BMJ Open. 2013;3:e03950.
23. van Middelkoop M, Dziedzic KS, Doherty M, et al. Individual patient data meta-analysis of trials investigating the effectiveness of intraarticular glucocorticoid injections in patients with knee or hip osteoarthritis: an OA Trial Bank protocol for a systematic review. Syst Rev. 2013;2:54.

24. van Middelkoop M, Arden NK, Atchia I, et al. The OA Trial Bank: metaanalysis of individual patient data from knee and hip osteoarthritis trials show that patients with severe pain exhibit greater benefit from intraarticular glucocorticoids. Osteoarthritis Cartilage. 2016;24:1143-1152.

25. Li G, Cook DJ, Levine MA, et al. Competing risk analysis for evaluation of dalteparin versus unfractionated heparin for venous thromboembolism in medical-surgical critically ill patients. Medicine. 2015;94:e1479.

26. Kahan BC, Morris TP. Analysis of multicentre trials with continuous outcomes: when and how should we account for centre effects? Stat Med. 2013;32:1136-1149.

27. Ma J, Thabane L, Kaczorowski J, et al. Comparison of Bayesian and classical methods in the analysis of cluster randomized controlled trials with a binary outcome: the Community Hypertension Assessment Trial (CHAT). BMC Med Res Methodol. 2009;9:1.

28. Pogue J, Devereaux PJ, Thabane L, Yusuf S. Designing and analyzing clinical trials with composite outcomes: consideration of possible treatment differences between the individual outcomes. PLoS One. 2012;7:e34785.

29. Samaan Z, Mbuagbaw L, Kosa D, et al. A systematic scoping review of adherence to reporting guidelines in health care literature. J Multidiscip Healthc. 2013;6:169-188.

30. Tunis AS, McInnes MD, Hanna R, Esmail K. Association of study quality with completeness of reporting: have completeness of reporting and quality of systematic reviews and meta-analyses in major radiology journals changed since publication of the PRISMA statement? Radiology. 2013;269:413-426.

31. Li G, Thabane L, Cook DJ, et al. Risk factors for and prediction of mortality in critically ill medical-surgical patients receiving heparin thromboprophylaxis. Ann Intensive Care. 2016;6:18.

32. Li G, Cook DJ, Thabane L, et al. Risk factors for mortality in patients admitted to intensive care units with pneumonia. Respir Res. 2016;17:80.

33. Cook D, McDonald E, Smith O, et al. Co-enrollment of critically ill patients into multiple studies: patterns, predictors and consequences. Crit Care. 2013;17:R1.

34. Hooven FH, Adachi JD, Adami S, et al. The Global Longitudinal Study of Osteoporosis in Women (GLOW): rationale and study design. Osteoporos Int. 2009;20:1107-1116.

35. Li G, Ioannidis G, Pickard L, et al. Frailty index of deficit accumulation and falls: data from the Global Longitudinal Study of Osteoporosis in Women (GLOW) Hamilton cohort. BMC Musculoskelet Disord. 2014;15:185.

36. Li G, Thabane L, Ioannidis G, Kennedy C, Papaioannou A, Adachi JD. Comparison between frailty index of deficit accumulation and phenotypic model to predict risk of falls: data from the Global Longitudinal Study of Osteoporosis in Women (GLOW) Hamilton cohort. PLoS One. 2015;10:e120144.

37. Li G, Thabane L, Papaioannou A, Adachi JD. Comparison between frailty index of deficit accumulation and fracture risk assessment tool (FRAX) in prediction of risk of fractures. Bone. 2015;77:107-114.

38. Li G, Papaioannou A, Thabane L, Cheng J, Adachi JD. Frailty change and major osteoporotic fracture in the elderly: data from the global longitudinal study of osteoporosis in women 3-year Hamilton cohort. J Bone Miner Res. 2016;31:718-724.

39. Mbuagbaw L, Thabane L. How to set-up a long-distance mentoring program: a framework and case description of mentorship in HIV clinical trials. J Multidiscip Healthc. 2013;6:17-23.

40. Thabane L, Walter SD, Hanna S, Goldsmith CH, Pullenayegum E. Developing a biostatistical collaboration course in a health science research methodology program. J Stat Educ. 2008;16:1-16.

41. Pullenayegum EM, Thabane L. Teaching Bayesian Statistics in a Health Research Methodology Program. J Stat Educ. 2009;17:n3. 
42. Thabane L, Wells G, Cook R, et al. Canadian-led capacity-building in biostatistics and methodology in cardiovascular and diabetes trials: the CANNeCTIN Biostatistics and Methodological Innovation Working Group. Trials. 2011;12:48.

43. Thabane L, Ma J, Chu R, et al. A tutorial on pilot studies: the what, why and how. BMC Med Res Methodol. 2010;10:1.

44. Sackett DL. On the determinants of academic success as a clinicianscientist. Clin Invest Med. 2001;24:94-100.

45. Sackett DL. Clinician-trialist rounds: 3 . Priority setting for academic success. Clin Trials. 2011;8:235-237.

46. Straus SE, Sackett DL. Clinician-trialist rounds: 7. Mentoring: why every clinician-trialist needs to get mentored. Clin Trials. 2011;8:765-767.

47. Straus SE, Sackett DL. Clinician-trialist rounds: 8. Mentoring-part 2: the structure and function of effective mentoring linkage, resources, and academic opportunities. Clin Trials. 2012;9:128-131.
48. Miracle VA, King KC. Presenting research: effective paper presentations and impressive poster presentations. Appl Nurs Res. 1994;7: 147-151.

49. Glew RH, Challa AK, Gopalan V. Training in scientific manuscript writing. Curr Sci. 2014;107:1386.

50. Guyatt GH, Brian Haynes R. Preparing reports for publication and responding to reviewers' comments. J Clin Epidemiol. 2006;59: 900-906.

51. CONSORT statement [homepage on the Internet]. Ottawa: The CONSORT Group; 2013. Available from: http://www.consort-statement.org/. Accessed April 15, 2017.

52. EQUATOR network [homepage on the internet]. London: the Centre for Statistics in Medicine, NDORMS, University of Oxford; 2016. Available from http://www.equator-network.org/. Accessed April 15, 2017. 


\section{Supplementary materials}

Table SI More examples of studies with published protocols and results papers

\begin{tabular}{|c|c|c|c|}
\hline Study design & Study name & Protocol paper* & Results paper* \\
\hline Mixed methods research studies & N/A & Mbuagbaw, 2013' & Mbuagbaw, $2014^{2}$ \\
\hline Pilot randomized controlled trials & PROSPECT & Johnstone, $2015^{3}$ & Cook, $2016^{4}$ \\
\hline Reporting guideline development & $\begin{array}{l}\text { CONSORT extension for } \\
\text { pilot trials }\end{array}$ & Thabane, $2016^{5}$ & Eldridge, $2016^{6}$ \\
\hline \multirow[t]{2}{*}{ Quality of reporting assessment for published studies } & $\mathrm{N} / \mathrm{A}$ & Madden, $2016^{7}$ & Study ongoing \\
\hline & N/A & Thabane, $2015^{8}$ & Study ongoing \\
\hline Statistical analysis plans & HONEYPOT study & Johnson, $2009^{9}$ & Pascoe, $2013^{10}$ \\
\hline
\end{tabular}

Note: *Expressed as first author, publication year reference number.

Abbreviations: PROSPECT, Prevention of Severe Pneumonia and Endotracheal Colonization Trial; CONSORT, Consolidated Standards of Reporting Trials; HONEYPOT study, Antibacterial honey for the prevention of catheter-associated infections in peritoneal dialysis patients; N/A, not applicable.

Table S2 More examples of sharing academic process or experience

\begin{tabular}{|c|c|}
\hline Strategies & Example study reference* \\
\hline Experiences in mentorship relationships & Odueyungbo, $2012^{11}$ \\
\hline \multirow[t]{3}{*}{ Capacity building and skill development } & Thabane, $2007^{12}$ \\
\hline & Thabane, $2008^{13}$ \\
\hline & Machekano, $2015^{14}$ \\
\hline \multirow[t]{6}{*}{ Challenges, lessons, or suggestions during research or collaborations } & Thabane, $2009^{15}$ \\
\hline & Mbuagbaw, 201 I ${ }^{16}$ \\
\hline & Ye, $2011^{17}$ \\
\hline & Thabane, $2010^{18}$ \\
\hline & Thabane, $2013^{19}$ \\
\hline & $\mathrm{Li}, 2016^{20}$ \\
\hline
\end{tabular}

Note: *Expressed as first author, publication year reference number.

\section{References}

1. Mbuagbaw L, Ongolo-Zogo P, Thabane L. Investigating community ownership of a text message programme to improve adherence to antiretroviral therapy and provider-client communication: a mixed methods research protocol. BMJ Open. 2013; 3:e002816.

2. Mbuagbaw L, Bonono-Momnougui RC, Thabane L, Kouanfack C, Smieja M, Ongolo-Zogo P. A framework for community ownership of a text messaging programme to improve adherence to antiretroviral therapy and client-provider communication: a mixed methods study. BMC Health Serv Res. 2014;14:441.

3. Johnstone J, Meade M, Marshall J, et al. Probiotics: prevention of severe pneumonia and endotracheal colonization trial - PROSPECT: protocol for a feasibility randomized pilot trial. Pilot Feasibility Stud. 2015;1:19.

4. Cook DJ, Johnstone J, Marshall JC, et al. Probiotics: prevention of severe pneumonia and endotracheal colonization trial - PROSPECT: a pilot trial. Trials. 2016;17:377.

5. Thabane L, Hopewell S, Lancaster GA, et al. Methods and processes for development of a CONSORT extension for reporting pilot randomized controlled trials. Pilot Feasibility Stud. 2016;2:25.

6. Eldridge SM, Chan CL, Campbell MJ, et al. CONSORT 2010 statement: extension to randomised pilot and feasibility trials. $B M J$ 2016;355:i5239.

7. Madden K, Arseneau E, Evaniew N, Smith CS, Thabane L. Reporting of planned statistical methods in published surgical randomised trial protocols: a protocol for a methodological systematic review. BMJ Open. 2016;6(6):e011188.

8. Thabane A, Dennis BB, Gajic-Veljanoski O, Paul J, Thabane L. Reporting quality of stepped wedge design randomized trials: a systematic review protocol. Clin Epidemiol. 2016;8:261-266.
9. Johnson DW, Clark C, Isbel NM, et al. The honeypot study protocol: a randomized controlled trial of exit-site application of medihoney antibacterial wound gel for the prevention of catheter-associated infections in peritoneal dialysis patients. Perit Dial Int. 2009;29:303-309.

10. Pascoe EM, Lo S, Scaria A, et al. The HONEYPOT randomized controlled trial statistical analysis plan. Perit Dial Int. 2013;33(4): 426-435.

11. Odueyungbo A, Thabane L. Mentoring in biostatistics: some suggestions for reform. J Multidiscip Healthc. 2012;5:265.

12. Thabane L, Thabane M, Goldsmith $\mathrm{CH}$. Mentoring young statisticians: facilitating the acquisition of important career skills. Afr Stat J. 2007;4:123-136.

13. Thabane L, Walter SD, Hanna S, Goldsmith $\mathrm{CH}$, Pullenayegum E. Developing a biostatistical collaboration course in a health science research methodology program. J Stat Educ. 2008;16:1-16.

14. Machekano R, Young T, Rusakaniko S, et al. The Africa Center for Biostatistical Excellence: a proposal for enhancing biostatistics capacity for sub Saharan Africa. Stat Med. 2015;34:3481-3489.

15. Thabane L, Thomas T, Ye C, Paul J. Posing the research question: not so simple. Can J Anesth. 2009;56:71-79.

16. Mbuagbaw L, Thabane L, Ongolo-Zogo P, Lang T. The challenges and opportunities of conducting a clinical trial in a low resource setting: the case of the Cameroon Mobile Phone SMS (CAMPS) trial, an investigator initiated trial. Trials. 2011;12:145.

17. Ye C, Giangregorio L, Holbrook A, Pullenayegum E, Goldsmith CH, Thabane L. Data withdrawal in randomized controlled trials: defining the problem and proposing solutions: a commentary. Contemp Clin Trials. 2011;32:318-322.

18. Thabane L, Ma J, Chu R, et al. A tutorial on pilot studies: the what, why and how. BMC Med Res Methodol. 2010;10:1. 
19. Thabane L, Mbuagbaw L, Zhang S, et al. A tutorial on sensitivity analyses in clinical trials: the what, why, when and how. BMC Med Res Methodol. 2013;13:92.
20. Li G, Taljaard M, Van den Heuvel ER, et al. An introduction to multiplicity issues in clinical trials: the what, why, when and how. Int $J$ Epidemiol. 2017;46:746-755.

\section{Publish your work in this journal}

The Journal of Multidisciplinary Healthcare is an international, peerreviewed open-access journal that aims to represent and publish research in healthcare areas delivered by practitioners of different disciplines. This includes studies and reviews conducted by multidisciplinary teams as well as research which evaluates the results or conduct of such teams or health care processes in general. The journal covers a very wide range of areas and welcomes submissions from practitioners at all levels, from all over the world. The manuscript management system is completely online and includes a very quick and fair peer-review system. Visit http://www.dovepress.com/ testimonials.php to read real quotes from published authors. 